Research Article

\title{
Eye-Tracking Analysis for Emotion Recognition
}

\author{
Paweł Tarnowski (D), Marcin Kołodziej, Andrzej Majkowski, and Remigiusz Jan Rak \\ Institute of Theory of Electrical Engineering, Measurement, and Information Systems, Warsaw University of Technology, \\ Warsaw 00-662, Poland \\ Correspondence should be addressed to Paweł Tarnowski; tarnowsp@ee.pw.edu.pl
}

Received 11 December 2019; Revised 23 July 2020; Accepted 3 August 2020; Published 1 September 2020

Academic Editor: Amparo Alonso-Betanzos

Copyright (c) 2020 Paweł Tarnowski et al. This is an open access article distributed under the Creative Commons Attribution License, which permits unrestricted use, distribution, and reproduction in any medium, provided the original work is properly cited.

\begin{abstract}
This article reports the results of the study related to emotion recognition by using eye-tracking. Emotions were evoked by presenting a dynamic movie material in the form of 21 video fragments. Eye-tracking signals recorded from 30 participants were used to calculate 18 features associated with eye movements (fixations and saccades) and pupil diameter. To ensure that the features were related to emotions, we investigated the influence of luminance and the dynamics of the presented movies. Three classes of emotions were considered: high arousal and low valence, low arousal and moderate valence, and high arousal and high valence. A maximum of $80 \%$ classification accuracy was obtained using the support vector machine (SVM) classifier and leaveone-subject-out validation method.
\end{abstract}

\section{Introduction}

1.1. Motivation. Emotions play a very important role in everyone's life. People feel emotions during everyday tasks, during interpersonal communication, when making decisions, learning, or during cognitive activities. For several years, a large increase in the number of studies on methods of automatic recognition of emotions has been observed. This is due to the increasing number and widespread use of electronic devices such as smartphones, tablets, and computers. The issue of recognizing emotions is an interdisciplinary problem and includes computer science, psychology, and cognitive science. The development of effective methods for recognizing emotions can not only improve the interaction of people with machines, but also contribute to the development of other areas such as psychology, medicine [1], education [2], and entertainment [3].

1.2. State of the Art. Automatic recognition of emotions in medicine can be used to diagnose and treat diseases such as posttraumatic stress disorder [4] and depression [5]. It is also used in diseases such as autism or Asperger's syndrome $[6,7]$. Another application of emotion recognition is education and supporting distance learning. In [8], a speech signal was used to recognize students' emotions during the lesson. In [9], a tool is presented to visualize the degree of attention and involvement of students, using the measurement of electrodermal skin activity. Emotion recognition systems can improve teacher-student interaction and, as a result, lead to better learning results. Currently, due to the rapidly growing number of multimedia materials appearing on the Internet, numerous studies are carried out related to their automatic labeling. Movies or music is often designed to evoke specific emotions; hence the automatically generated labels should contain information about the emotional characteristics of the material [10]. In [11], an EEG signal was used to label movies in terms of evoked emotions. To recognize emotions, the features of the signal registered for a group of people watching the same set of movies were used. Similar studies are described in $[12,13]$ using, in addition to the EEG signal, facial expressions.

Emotions are felt by a person as certain reactions of the body to a given situation or stimulus. The occurrence of these reactions is the basis for the operation of systems for recognizing the emotional state of a person. Facial expressions $[12,14,15]$, speech signal recordings [16, 17], and brain activity and changes in other human physiological 
parameters are subject to testing. In the case of brain activity, signals from the central nervous system are recorded using electroencephalography (EEG) $[12,14,15]$, as well as medical magnetic resonance imaging (fMRI) [18]. Other physiological signals used include: electrocardiography (ECG) [19-21], electromyography (EMG) [22], electrodermal activity (EDA) [19, 20, 23], heart rate [24-26], respiration rate and depth [24, 27], and arterial pressure [24]. Eye-tracking [28-32] and pupil width [33-36] are also used to recognize emotions.

Interest in eye movement research dates back to the nineteenth century when the method of direct observation was used. Then, invasive eye-trackers were constructed using a special type of lens placed on the eyeball. At present, noncontact devices, which use infrared cameras, are most commonly used for eye-tracking. The development of such devices increased the popularity of eye movement research among scientists from many different fields. Eye-tracking research is applied in areas such as cognitive psychology, neuropsychology, usability testing, or marketing [37]. The eyeball movement is examined while viewing the presented materials, e.g., photos or movies, and while reading, playing, browsing the web, or using graphical user interfaces. Few attempts have also been made to use eye-tracking to recognize emotions [32]. In [28], features associated with eye movements were used to recognize three classes of emotions. The classification accuracy obtained was $77.8 \%$. In [38], for three classes of emotions recognized using eye-tracking methods, the classification accuracy was $68.8 \%$. In [30], the use of features such as fixation duration, amplitude and duration of saccades, and blinking frequency for classification of four classes of emotions was described, achieving $67.82 \%$ accuracy. In all mentioned studies, emotions were evoked by presenting movies.

The eye-tracking often includes measuring the diameter of the pupil, which is responsible for regulating the amount of light falling on the retina. In addition to light intensity, the pupil response is also influenced by other factors. These factors include the emotions experienced by oneself $[39,40]$. To date, studies have shown that the pupil diameter increases when feeling excited [34, 36]. In [29], an attempt to recognize three classes of emotions (positive, negative, and neutral) evoked by movies using pupil width was presented. Classification accuracy of 58.9\% was achieved. The study [41] describes research on recognizing emotions caused by playing a video game. A set of features related to pupil width were used for the classification. For three classes of emotions, the classification accuracy achieved was $76 \%$ and $61.4 \%$, respectively, for the arousal and the valence scales. The work [35] presents the use of pupillometry to measure emotional arousal in videos.

1.3. The Aim of This Paper. The purpose of the presented research is to analyze whether it is possible to recognize emotions by using eye-tracking signal. Emotions have been caused by the presentation of video material. For the present research, an experiment was designed during which a group of 30 participants watched a set of 21 fragments of movies intended to evoke emotions. During movie presentation, eye-tracking data were recorded. As features, we used eyemovement specific elements such as fixations and saccades. Features related to the pupil diameter were also calculated. We recognized three classes of emotions: high arousal and low valence, low arousal and moderate valence, and high arousal and high valence. Three classifiers were tested: SVM, LDA, and $k$-NN. The leave-one-subject-out method was used to assess the quality of the classification. Individual, successive stages of the research were the following:

(i) acquisition of eye-tracking data while evoking emotions

(ii) performing signal preprocessing

(iii) removal of the effect of luminance on pupil width

(iv) calculation of eye-tracking features related to eye movements and pupil width

(v) classification of emotions using SVM, LDA, and $k$ NN

Video material is characterized by fast scene changes and moving objects. The dynamics of the movies can greatly influence the eye-tracking features and the possibility to use them for emotion recognition. Innovative in our research is that we examined the effects of the dynamics of movies on classification accuracy. Further, when measuring the pupil diameter, the impact of luminance and lighting conditions is very important. The effect of luminance on pupil width was removed using linear regression.

\section{Materials and Methods}

2.1. Evoking Emotions. An important element of the study was evoking emotions in participants of the experiment. Emotions are most often caused by the presentation of visual stimuli (images and movies) [34, 38, 42, 43] and sound stimuli (music and sounds) $[36,44]$. In our experiment, emotions were evoked by a video presentation on a monitor screen. The presented material comprised short movies along with the soundtrack. Twenty-one movies were used in the experiment. They elicited six basic emotions, as defined in [45], that is, happiness, sadness, anger, surprise, disgust, and fear. Several other movies were selected to be as neutral as possible and did not cause any of the above emotions. The selection of movies that cause a specific emotion is a difficult task. Therefore, a survey was conducted to ensure that a movie affects the examined participant in the intended way. The questionnaire was designed to examine the feelings and emotions experienced after seeing individual pieces of movies. Each participant was asked the following 3 questions about each movie:

(1) Evaluate the emotions you experienced while watching a movie from 1 (unpleasant-low valence) to 9 (pleasant-high valence).

(2) Rate the intensity of the emotions you experienced while watching a movie from 1 (nonstimulating-low arousal) to 9 (highly stimulating-high arousal). 
(3) Choose the type of emotion you felt while watching the movie: neutral, happiness, sadness, anger, surprise, disgust, and fear. A participant also had the opportunity to describe his/her feelings.

The survey among the participants allowed creating a circumplex model of emotions $[46,47]$. In this model, the horizontal axis represents emotion valence, whereas the vertical axis represents arousal. Each emotion can be considered as a linear combination of these dimensions. Figure 1 shows the distribution of emotions caused by each movie (numbered from 1 to 21) used in the experiment on the valence-arousal plane. The distribution is based on the answers given by the participants in surveys.

The distribution of emotions resembles the shape of letter "V;" this is because movies that are very pleasant or very unpleasant are rated as stimulating at the same time. Neutral materials are generally rated as nonstimulating [48]. These tendencies are also reported in previous works $[49,50]$. We created three classes of emotions from the distribution of movies on the plane. Each class included two movies. Class $\mathrm{C} 1$ covered videos that were rated in surveys as very pleasant and very stimulating (high arousal and high valence). Class C2 included neutral, low stimulating movies (low arousal and moderate valence). Class C3 contained very unpleasant and highly stimulating movies (high arousal and high valence). Only these 6 movies were further considered in the experiment. Table 1 presents the mean and median values of valence and arousal assigned to the selected movies by the participants. Movies \#2 and \#12 presented dancing women with rhythmic music. Movie \#3 presented a bird's eye view of the highway with calm music. Movie \#18 presented the weather forecast. Movie \#13 included scenes of violence against a woman, while movie \#20 showed amputation of a finger.

2.2. Participants of the Experiment. A specific group of people selected based on age, gender, and education were invited to participate in the experiment. Participants were recruited through an advertisement on the website of Faculty of Electrical Engineering, Warsaw University of Technology. Finally, 30 volunteers took part in the experiment. All participants were male third-year students with an average age of $21.25 \pm 0.74$ years. They were informed about the overall purpose and organization of the experiment and agreed to participate in it. Volunteers were not paid for participating in the experiment.

2.3. Experimental Procedure. The experiment was carried out in a specially prepared room, and its temperature and lighting were the same for all participants. The test stand consisted of a PC, EyeTribe eye-tracker, and two Creative Inspire T10 speakers. The experiment always started in the morning between 7:00 and 8:00 AM. The participants were asked to take a comfortable position on the armchair that supported their hands and back in front of the computer monitor. Next, eye-tracker calibration was performed. The person supervising the experiment then left the room, and the participant alone watched the video material. The movies shown had a soundtrack. The sound volume was identical for all participants. We developed our own software responsible for controlling the course of the experiment, which enabled simultaneous display of movies and eye-tracking signal acquisition [51]. The movie prepared for the experiment lasted $8 \mathrm{~min} 10 \mathrm{~s}$. Different clips of the video material that evoked different emotions were separated by a sequence of $5 \mathrm{~s}$ of black screen and $5 \mathrm{~s}$ of colour animated curves that resembled a screen saver. The break between the videos was intended to curtail the previous emotions and prepare the participant for the next part of the movie. Each participant watched the same video material with a random order of 21 short movies associated with particular emotions. Figure 2 shows a typical sequence of the presented movies. After the video material was played, each participant had to answer the questions in a survey on emotions caused by movies.

2.4. Acquisition of Eye-Tracking Data. The EyeTribe [52] optical eye-tracker was used to register oculographic signal. The EyeTribe calculates a pair of $(X, Y)$ coordinates of the user's gaze point with respect to a screen the user is looking at. The coordinates are expressed in pixels. Accuracy of calculating user's eye gaze coordinates is approximately $1^{\circ}$. Such an accuracy can be obtained after proper calibration of the device. Calibration using 9 measurement points was performed for each participant. The EyeTribe also provides information about the occurrence of eye fixations and measures the pupil diameter of both eyes. The eye-tracking signal was registered with a frequency of $60 \mathrm{~Hz}$.

2.5. Signal Preprocessing. Some of the participants' eye movement information was lost, for example, during blinking or during other eye or head movements. Data from a participant were rejected completely when the number of lost samples was greater than $25 \%$. Thus, data recorded for 5 participants were rejected. Further analysis was performed on data recorded for the remaining 25 participants. Samples, lost due to, for example, blinking, were supplemented by linear interpolation. The signal was also filtered with $4^{\text {th }}$ order low-pass Butterworth filter with a cutoff frequency equal to $5 \mathrm{~Hz}$. Filtration was designed to eliminate highfrequency noise. Figure 3(a) shows the recorded eyetracking signal ( $X$ and $Y$ coordinates of the gaze point expressed in pixels): (B) interpolated signal and (C) signal after low-pass filtering.

2.6. Feature Extraction. The analysis of eye-tracking data involved calculating the features of the signal that depends on the emotional content of a movie. Raw data points were processed into eye movements such as fixations and saccades [37]. There are many algorithms to calculate fixation; these algorithms are based on the speed of eye movement (I-VT) and data scattering (I-DT) or are related to areas of interest (AOI-I) [53]. The EyeTribe eye-tracker has a built-in algorithm that classifies a given sample of a registered signal and informs one whether a given signal sample represents a 


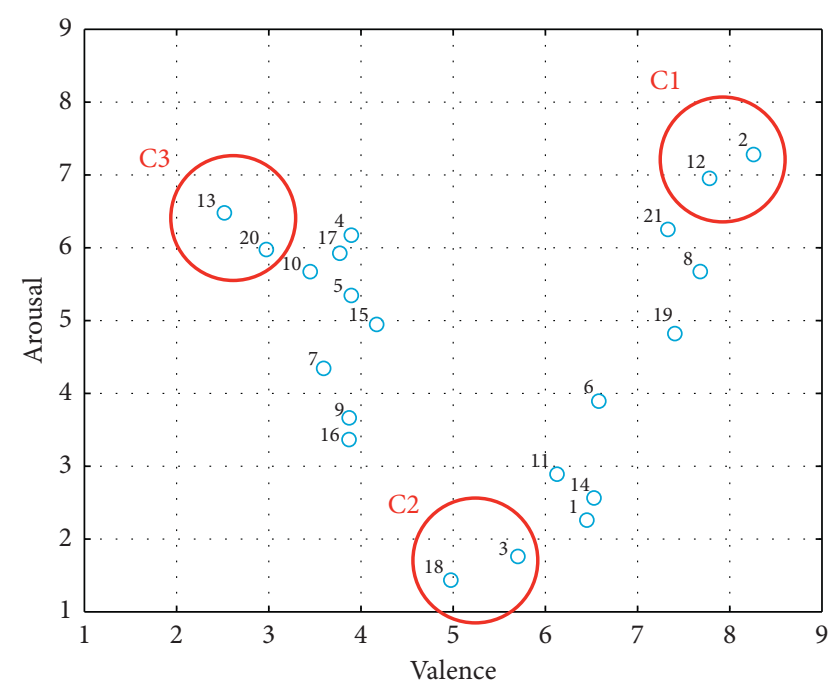

FIGURE 1: Distribution of emotions caused by movies (numbered from 1 to 21 ) on the valence-arousal plane along with created classes (marked red).

TABLE 1: Evaluation of the selected movies.

\begin{tabular}{lcccccc}
\hline Class & $\begin{array}{c}\text { Video } \\
\text { numbers }\end{array}$ & $\begin{array}{c}\text { Duration } \\
(\mathrm{s})\end{array}$ & \multicolumn{2}{c}{ Arousal } & \multicolumn{2}{c}{ Valence } \\
& Mean & Median & Mean & Median \\
\hline \multirow{2}{*}{ C1 } & 2 & 10 & 7.3 & 8 & 8.1 & 9 \\
& 12 & 13 & 7.2 & 7.5 & 7.9 & 8.5 \\
\hline \multirow{2}{*}{ C2 } & 3 & 5 & 1.9 & 1 & 5.6 & 5 \\
& 18 & 10 & 1.3 & 1 & 4.8 & 5 \\
\hline \multirow{2}{*}{ C3 } & 13 & 10 & 6.6 & 6.5 & 2.8 & 2 \\
& 20 & 10 & 6.4 & 6 & 3.3 & 2.5 \\
\hline
\end{tabular}

fixation. Figure 4 shows a fragment of the registered visual coordinates along with the marked fixations. There are 6 fixations identified by the eye-tracker in the shown signal fragment.

Features were calculated in time windows covering each of the movie fragments. Fixations were used to calculate features such as the number of fixations, mean value, variance, skewness, and kurtosis of duration of fixations. The fixation durations were expressed as the number of samples. Based on fixations, the overall fixation vector (OFV) was also calculated. This feature considers the number, position, and duration of fixations. It is described by the following $[37,54]$ :

$$
\mathrm{OFV}=\sum_{i=1}^{N} t_{i} \mathbf{v}_{i}
$$

where $\mathbf{v}_{\mathbf{i}}=\left(x_{i}-x_{c}, y_{i}-y_{c}\right)$ is a vector with an origin in the center of the screen $\left(x_{c}, y_{c}\right)$ and the end at the fixation point $\left(x_{i}, y_{i}\right), t_{i}$ is the duration of the $i$-th fixation. Pairs $\left(x_{c}, y_{c}\right)$ and $\left(x_{i}, y_{i}\right)$ are coordinates, expressed as pixels, of the center of the screen and the fixation point, respectively. Mean value, variance, skewness, and kurtosis of amplitudes and the duration of the saccades were also calculated. The duration of saccades was expressed as the number of samples, while amplitude was expressed as pixels. Some features such as the number of fixations depend on the duration of the movie for which they were calculated. To normalize these features, they were divided by the length of the movie expressed as the number of samples. Therefore, these features were not correlated with the length of the analysed part of the movie.

The pupil diameter enables the detection of changes in the intensity of human emotions at a given moment $[34,36]$. In addition to eye movement, the EyeTribe eyetracker allows one to measure the pupil diameter. Mean value, variance, skewness, and kurtosis of the pupil diameter, which were measured during watching a given movie, were calculated. Table 2 lists the calculated features. The features were grouped according to the type of eye movement.

2.7. Classification. The classification was performed using 18 eye-tracking features taken from 25 participants. The features were calculated for a whole movie associated with one type of emotion. Each emotion was evoked by two different movies. Features were standardized by centering them to have a mean value equal to zero and by scaling them to have standard deviation equal to one ( $z$-score) for each participant. Therefore, for 25 participants, the total number of examples related to one emotion was 50 ( 2 movies $\times 25$ volunteers). Hence, to classify emotions in pairs and in triplets, the number of examples was 100 and 150, respectively.

As classifiers, we used linear discriminant analysis (LDA), support vector machine with a square kernel (Quadratic SVM), and the nearest neighbour classifier ( $k$ $\mathrm{NN}$ ) with $k=11$ [55]. The parameters of individual classifiers were selected experimentally based on the accuracy of the classification. Classification was performed in the user-independent mode. Training dataset included examples from 24 participants and testing dataset included examples from 1 remaining participant so leave-one-out method was used for tests. The division of the data into training and testing sets was repeated 25 times. This enabled calculation of the average classification accuracy. Examples that were used in the training process were not used again during testing the classifier.

\section{Results}

Classification was performed for three pairs and for all three classes of emotions together. Classification accuracies achieved for each case are shown in Table 3. The highest accuracy of $80 \%$ of the classification of three classes of emotions was obtained using the SVM classifier. When recognizing emotions in pairs, it turned out that the easiest is to distinguish between classes $\mathrm{C} 1$ and $\mathrm{C} 2$. On the other hand, the most difficult is to distinguish between classes C2 and C3. Tables 4-6 show the confusion matrixes for the classification of all three classes for SVM, LDA, and $k$-NN classifiers, respectively.

Then, for each classifier parameters, precision $(\mathrm{P})$, recall (R), specificity (S), and F1-score (F1) were calculated for all three classes of emotions (Table 7). 


\begin{tabular}{|c|c|c|c|c|}
\hline Break & $\begin{array}{l}\text { Emotion } \\
\text { elicitation }\end{array}$ & Break & $\begin{array}{l}\text { Emotion } \\
\text { elicitation }\end{array}$ & Questionnaire \\
\hline 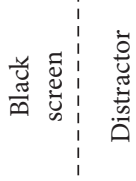 & 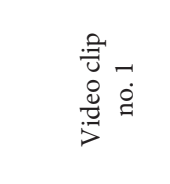 & 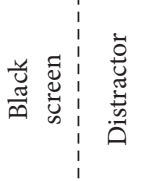 & 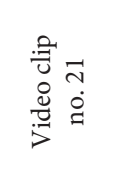 & 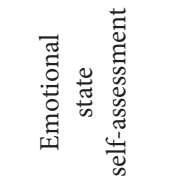 \\
\hline \begin{tabular}{l|l}
$5 \mathrm{~s}$ & $5 \mathrm{~s}$
\end{tabular} & $5-71.7 s$ & \begin{tabular}{|l|l}
$5 \mathrm{~s}$ & $5 \mathrm{~s}$
\end{tabular} & $5-71.7 s$ & Time \\
\hline
\end{tabular}

FIgURE 2: Typical sequence of the presented movies.

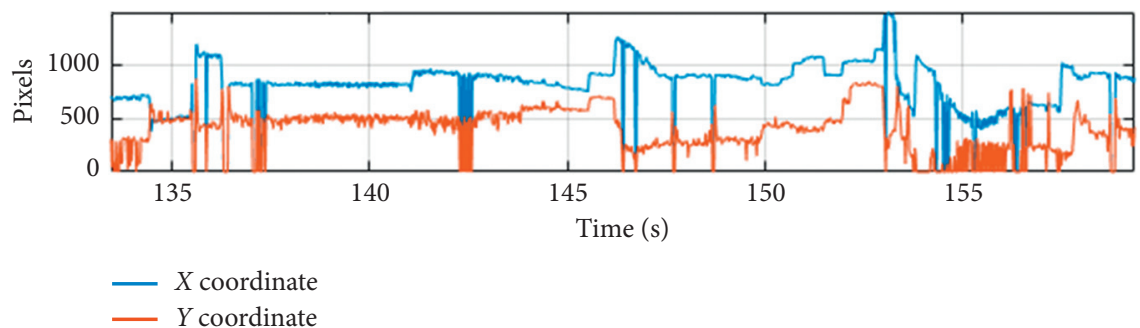

(a)

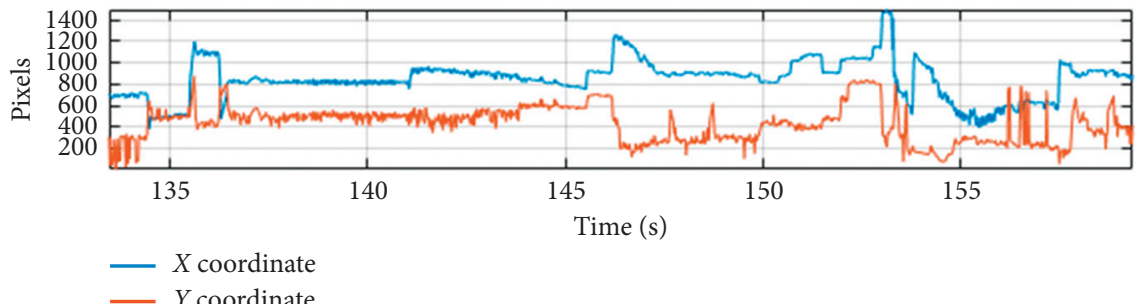

(b)

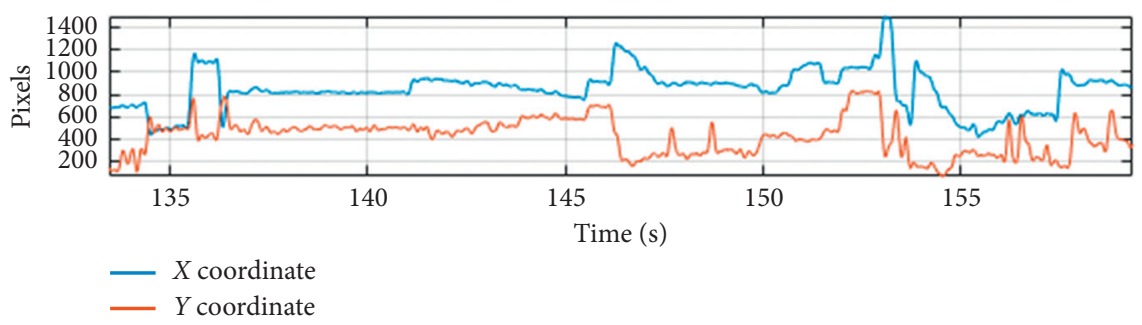

(c)

FIGURE 3: Preprocessing of eye-tracking signal: (a) pure signal, (b) signal after interpolation, and (c) signal after low-pass filtering.

Confusion matrixes analysis showed that the most classification errors appear for the C3 class. This is also confirmed by the values of precision, recall, and F1-score.

To answer the question of which features associated with eye movement can be a reliable indicator of emotions, statistical tests were performed. One-way analysis of variance (ANOVA) was used to determine whether features show a significant difference between the three classes. Before the ANOVA test was performed, it was checked whether the features satisfy normal distributions using Kolmogorov-Smirnov test. Distributions of the features were close to normal. Bonferroni post-hoc test for evaluating features among the groups was also made. Table 8 presents the results of the ANOVA and Bonferroni tests for each feature. The Bonferroni test showed that 9 features are suitable for distinguishing between classes $\mathrm{C} 1$ and $\mathrm{C} 2,8$ features for distinguishing between $\mathrm{C} 1$ and $\mathrm{C} 3$, and only 5 features for distinguishing between $\mathrm{C} 2$ and $\mathrm{C} 3$. This result is in line with previous observations; that is, the lowest classification accuracy was obtained when distinguishing between classes C2 and C3.

Mean values and standard deviations of all features for three classes of emotions are presented in Table 9.

The participant's pupil diameter is one of the statistically significant features. The pupil diameter reached the smallest value when experiencing neutral emotions (low arousal). The average pupil diameters for $\mathrm{C} 1, \mathrm{C} 2$, and C3 classes were $0.85,-0.74$, and -0.43 (z-score standardized), respectively. A similar trend was observed in other studies [28, 29, 34, 36]. Figure 5 shows the distribution of 


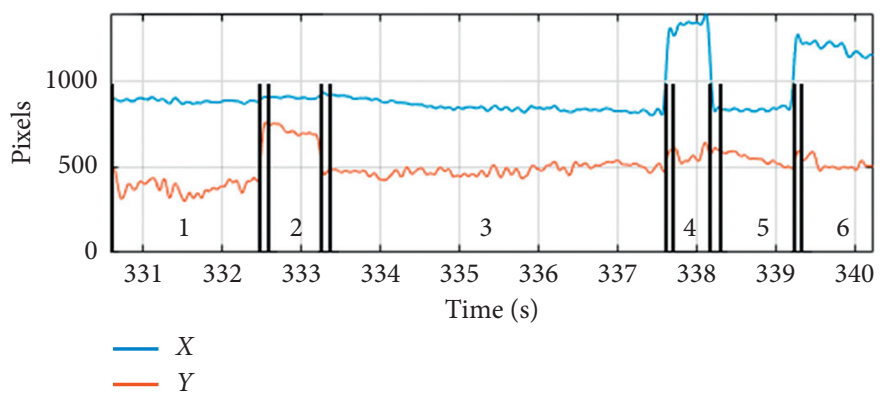

Figure 4: A fragment of an eye-tracking (fixations, saccades, and coordinates of the "sight").

TABLE 2: List of calculated features.

\begin{tabular}{lcc}
\hline Signal type & Features & No. \\
\hline \multirow{2}{*}{ Fixation } & Number of fixations & 1 \\
& Overall fixation vector & 2 \\
\hline \multirow{3}{*}{ Fixation duration } & Mean & 3 \\
& Variance & 4 \\
& Skewness & 5 \\
Saccade amplitude & Kurtosis & 6 \\
\hline & Mean & 7 \\
& Variance & 8 \\
Saccade duration & Skewness & 9 \\
& Kurtosis & 10 \\
\hline & Mean & 11 \\
Pupil diameter & Variance & 12 \\
& Skewness & 13 \\
& Kurtosis & 14 \\
\hline
\end{tabular}

TABle 3: Classification accuracies.

\begin{tabular}{lcccc}
\hline Classes & SVM & LDA & $k$-NN & Mean \\
\hline C1 vs. C2 vs. C3 & 0.80 & 0.73 & 0.65 & 0.73 \\
C1 vs. C2 & 0.92 & 0.85 & 0.86 & 0.88 \\
C1 vs. C3 & 0.83 & 0.84 & 0.75 & 0.81 \\
C2 vs. C3 & 0.75 & 0.78 & 0.70 & 0.74 \\
\hline
\end{tabular}

TABLE 4: Confusion matrix for SVM classifier.

\begin{tabular}{lccc}
\hline \multirow{2}{*}{ Predicted classes } & \multicolumn{3}{c}{ True classes } \\
& $\mathrm{C} 1$ & $\mathrm{C} 2$ & $\mathrm{C} 3$ \\
\hline $\mathrm{C} 1$ & 45 & 3 & 7 \\
$\mathrm{C} 2$ & 1 & 42 & 10 \\
$\mathrm{C} 3$ & 4 & 5 & 33 \\
\hline
\end{tabular}

the two features with the smallest $p$-values (average pupil diameter and average saccade amplitude) for the three classes of emotions.

\section{Discussion}

The obtained accuracy of emotion classification indicates that it is possible to distinguish emotions using eyetracking. However, when using eye-tracking and pupil
TABLE 5: Confusion matrix for LDA classifier.

\begin{tabular}{lccc}
\hline Predicted classes & \multicolumn{3}{c}{ True classes } \\
& C1 & C2 & C3 \\
\hline C1 & 41 & 6 & 7 \\
C2 & 1 & 34 & 5 \\
C3 & 8 & 10 & 38 \\
\hline
\end{tabular}

TABLE 6: Confusion matrix for $k$-NN classifier.

\begin{tabular}{lccc}
\hline \multirow{2}{*}{ Predicted classes } & \multicolumn{3}{c}{ True classes } \\
& C1 & C2 & C3 \\
\hline C1 & 48 & 13 & 20 \\
C2 & 0 & 33 & 13 \\
C3 & 2 & 4 & 17 \\
\hline
\end{tabular}

width, some additional factors that may affect the reliability of the results should be considered. The changes in the pupil diameter are largely dependent on the lighting conditions and the luminance of the movie $[29,56]$. The effect of luminance is so large that it is impossible to use the pupil diameter directly to recognize emotions without prior processing it. We have assumed that pupil changes are partly due to changes in the luminance of the movie and partly due to the experienced emotions. Therefore, the effect caused by the luminance of the movie had to be removed. For this purpose, the luminance of the movie was assessed by calculating for each of its frames the $\mathrm{V}$ component in the HSV colour space. The process of removing the movie luminance effect from the measurements of the pupil diameter is presented in Figure 6. Figure 6(a) shows a fragment of the luminance of the movie. Linear regression was used to model the relationship between the pupil diameter and luminance of a movie. For each participant, a linear model of the influence of luminance on the pupil diameter was calculated (coefficients $b_{0}, b_{1}$ ) using

$$
\left[\begin{array}{c}
y_{1} \\
y_{2} \\
\vdots \\
y_{n}
\end{array}\right]=\left[\begin{array}{cc}
1 & x_{1} \\
1 & x_{2} \\
\vdots & \vdots \\
1 & x_{n}
\end{array}\right]\left[\begin{array}{l}
b_{0} \\
b_{1}
\end{array}\right],
$$


Table 7: Precision, recall, specificity, and F1-score for all classifiers.

\begin{tabular}{|c|c|c|c|c|c|c|c|c|c|c|c|c|}
\hline \multirow{2}{*}{ Classes } & \multicolumn{4}{|c|}{ SVM } & \multicolumn{4}{|c|}{ LDA } & \multicolumn{4}{|c|}{$k-\mathrm{NN}$} \\
\hline & $\mathrm{P}$ & $\mathrm{R}$ & S & $\mathrm{F} 1$ & $\mathrm{P}$ & $\mathrm{R}$ & S & F1 & $\mathrm{P}$ & $\mathrm{R}$ & S & $\mathrm{F} 1$ \\
\hline $\mathrm{C} 1$ & 0.82 & 0.90 & 0.90 & 0.86 & 0.76 & 0.82 & 0.87 & 0.79 & 0.59 & 0.96 & 0.67 & 0.73 \\
\hline $\mathrm{C} 2$ & 0.79 & 0.84 & 0.89 & 0.81 & 0.85 & 0.68 & 0.94 & 0.76 & 0.72 & 0.66 & 0.87 & 0.69 \\
\hline C3 & 0.79 & 0.66 & 0.91 & 0.72 & 0.68 & 0.76 & 0.82 & 0.72 & 0.74 & 0.34 & 0.94 & 0.47 \\
\hline Mean & 0.80 & 0.80 & 0.90 & 0.80 & 0.76 & 0.75 & 0.88 & 0.76 & 0.68 & 0.65 & 0.83 & 0.63 \\
\hline
\end{tabular}

TABle 8: Results of ANOVA and Bonferroni post-hoc tests.

\begin{tabular}{|c|c|c|c|c|c|}
\hline \multirow{2}{*}{ Feature } & \multicolumn{2}{|c|}{ ANOVA } & \multicolumn{3}{|c|}{ Bonferroni multiple comparison test } \\
\hline & $p$ & $p<0.05$ & $\mathrm{C} 1$ vs. $\mathrm{C} 2 p$ & $\mathrm{C} 1$ vs. $\mathrm{C} 3 p$ & $\mathrm{C} 2$ vs. C3 $p$ \\
\hline Number of fixations & 0.32 & - & 1.00 & 1.00 & 0.40 \\
\hline Overall fixation vector & 0.64 & - & 1.00 & 1.00 & 1.00 \\
\hline Average duration of fixation & 0.01 & + & 1.00 & 0.01 & 0.02 \\
\hline Variance of fixation duration & 0.06 & - & 1.00 & 0.06 & 0.43 \\
\hline Skewness of fixation duration & 0.00 & + & 0.01 & 1.00 & 0.00 \\
\hline Kurtosis of fixation duration & 0.00 & + & 0.01 & 1.00 & 0.01 \\
\hline Average saccade amplitude & 0.00 & + & 0.00 & 0.65 & 0.00 \\
\hline Variance of the saccade amplitudes & 0.00 & + & 0.00 & 0.02 & 0.96 \\
\hline Skewness of the saccade amplitudes & 0.00 & + & 0.00 & 0.01 & 1.00 \\
\hline Kurtosis of the saccade amplitudes & 0.00 & + & 0.00 & 0.00 & 1.00 \\
\hline Average duration of saccades & 0.86 & - & 1.00 & 1.00 & 1.00 \\
\hline Variance of saccade durations & 0.56 & - & 1.00 & 0.89 & 1.00 \\
\hline Skewness of saccade durations & 0.00 & + & 0.00 & 0.01 & 0.99 \\
\hline Kurtosis of saccade durations & 0.00 & + & 0.00 & 0.00 & 0.71 \\
\hline Average pupil diameter & 0.00 & + & 0.00 & 0.00 & 0.35 \\
\hline Variance of the pupil diameter & 0.04 & + & 0.29 & 1.00 & 0.03 \\
\hline Skewness of the pupil diameter & 0.09 & - & 1.00 & 0.23 & 0.12 \\
\hline Kurtosis of the pupil diameter & 0.05 & - & 0.51 & 0.04 & 0.82 \\
\hline
\end{tabular}

TABLE 9: Mean values and standard deviations of all features for three classes of emotions.

\begin{tabular}{|c|c|c|c|c|c|c|c|}
\hline \multirow{2}{*}{ No. } & \multirow{2}{*}{ Feature } & \multicolumn{2}{|c|}{$\mathrm{C} 1$} & \multicolumn{2}{|c|}{$\mathrm{C} 2$} & \multicolumn{2}{|c|}{$\mathrm{C} 3$} \\
\hline & & Mean & Std. & Mean & Std. & Mean & Std. \\
\hline 1 & Number of fixations & -0.33 & 0.50 & -0.29 & 0.88 & 0.17 & 1.08 \\
\hline 2 & Overall fixation vector & -0.31 & 0.47 & -0.16 & 1.05 & 0.09 & 0.91 \\
\hline 3 & Average duration of fixation & 0.11 & 0.95 & -0.50 & 0.85 & 0.18 & 1.11 \\
\hline 4 & Variance of fixation duration & 0.17 & 1.04 & -0.42 & 0.63 & 0.15 & 1.14 \\
\hline 5 & Skewness of fixation duration & 0.18 & 0.76 & 0.03 & 1.23 & -0.13 & 1.00 \\
\hline 6 & Kurtosis of fixation duration & -0.31 & 0.48 & 1.00 & 1.00 & -0.11 & 0.90 \\
\hline 7 & Average saccade amplitude & -0.33 & 0.51 & 0.48 & 0.88 & 0.27 & 1.52 \\
\hline 8 & Variance of the saccade amplitudes & -0.37 & 0.60 & 0.17 & 0.96 & 0.17 & 0.88 \\
\hline 9 & Skewness of the saccade amplitudes & -0.44 & 0.59 & 0.24 & 0.85 & 0.11 & 0.86 \\
\hline 10 & Kurtosis of the saccade amplitudes & 0.12 & 0.93 & 0.09 & 1.26 & 0.01 & 1.07 \\
\hline 11 & Average duration of saccades & 0.15 & 1.07 & 0.08 & 1.22 & -0.08 & 0.97 \\
\hline 12 & Variance of saccade durations & 0.31 & 0.89 & -0.36 & 0.69 & -0.20 & 0.83 \\
\hline 13 & Skewness of saccade durations & 0.33 & 1.03 & -0.43 & 0.54 & -0.24 & 0.77 \\
\hline 14 & Kurtosis of saccade durations & 0.20 & 0.74 & 0.04 & 0.93 & 0.17 & 0.98 \\
\hline 15 & Average pupil diameter & 0.85 & 0.83 & -0.73 & 1.17 & -0.43 & 0.83 \\
\hline 16 & Variance of the pupil diameter & -0.04 & 0.68 & -0.31 & 0.69 & 0.11 & 1.04 \\
\hline 17 & Skewness of the pupil diameter & -0.06 & 0.66 & -0.01 & 0.72 & -0.35 & 1.06 \\
\hline 18 & Kurtosis of the pupil diameter & 0.09 & 0.66 & -0.13 & 0.81 & -0.31 & 0.89 \\
\hline
\end{tabular}

where $y$ is the average pupil diameter of both eyes recorded for a participant and $x$ is the luminance value calculated for a movie frame.

Then, the estimated value of the pupil diameter $y_{\text {est }}$ was calculated from

$$
y_{\mathrm{est}}=b_{0}+b_{1} x
$$

This caused the pupil diameter to be independent of luminance changes $((4))$. We assume that $y_{\text {emo }}$ depends on the felt emotions. 


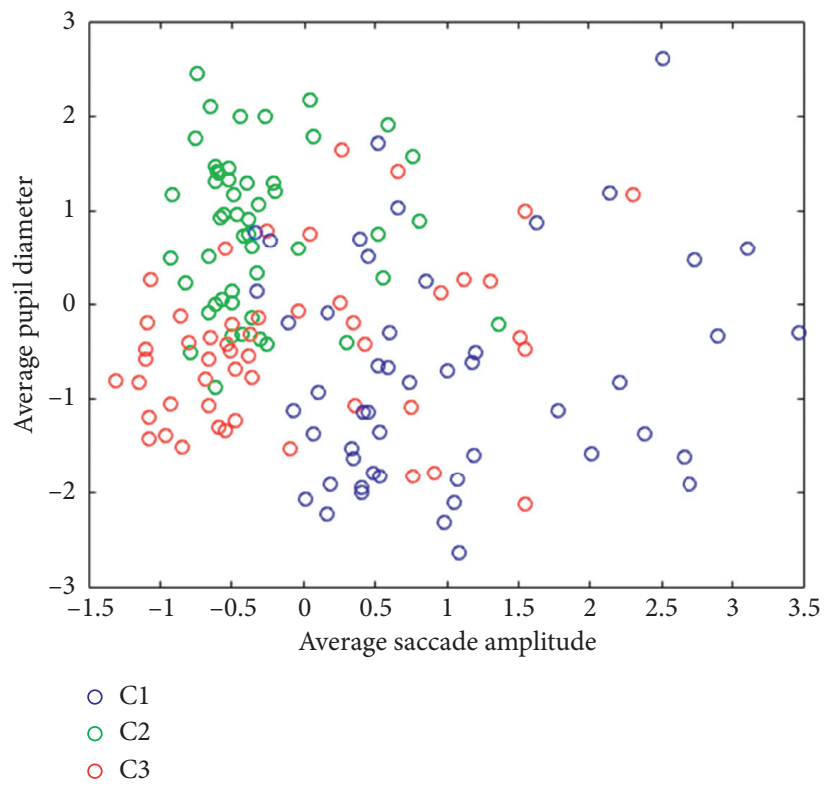

Figure 5: The distribution of the average pupil diameter and the average saccade amplitude for the three classes of emotions.
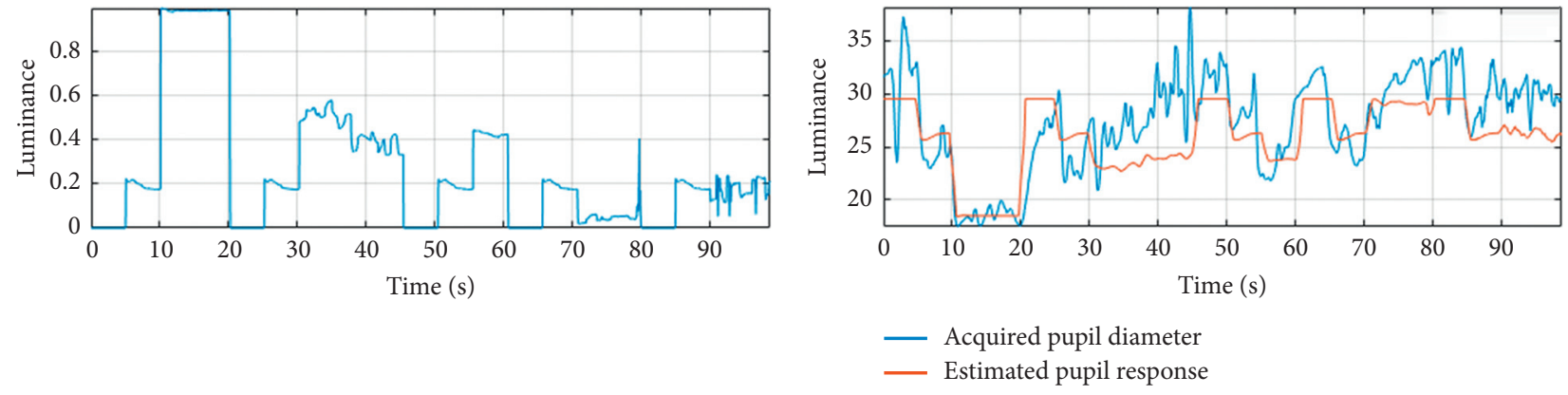

(a)

(b)

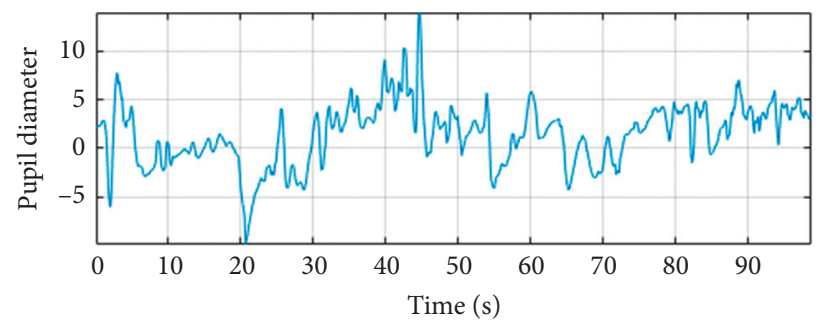

(c)

FIGURE 6: Removing the effect of movie luminance: (a) calculated luminance of a movie; (b) blue line, registered pupil diameter for a participant; red line, estimated pupil diameter (as a response for movie luminance); (c) pupil diameter after removing the luminance effect.

$$
y_{\mathrm{emo}}=y-y_{\mathrm{est}}
$$

Figure 6(b) shows the registered pupil diameter value for participant S06 and its estimated (as a response for movie luminance) value $y_{\text {est }}=29.84-11.23 x$. Figure 6(c) shows the pupil diameter of the same participant after removing the effect of luminance. The mean correlation between the pupil diameter and luminance for all volunteers was -0.49 . This value was reduced to 0.006 after subtracting the estimated luminance effect.
We also carried out additional tests to assess whether the method of removing the influence of luminance is sufficiently effective. First, we tested the approach taken from a prior publication [23], in which neighbourhood of the gaze position was used to calculate the luminance, instead of the average luminance of the whole frame. The neighbourhood had a width of 2 degrees (of visual angle) around the gaze point, which corresponds to a circle with a diameter of 68 pixels (distance from the monitor $60 \mathrm{~cm}$, resolution $1920 \times 1080$ pixels). Sample frames from movies with marked gaze positions are shown in Figure 7. 


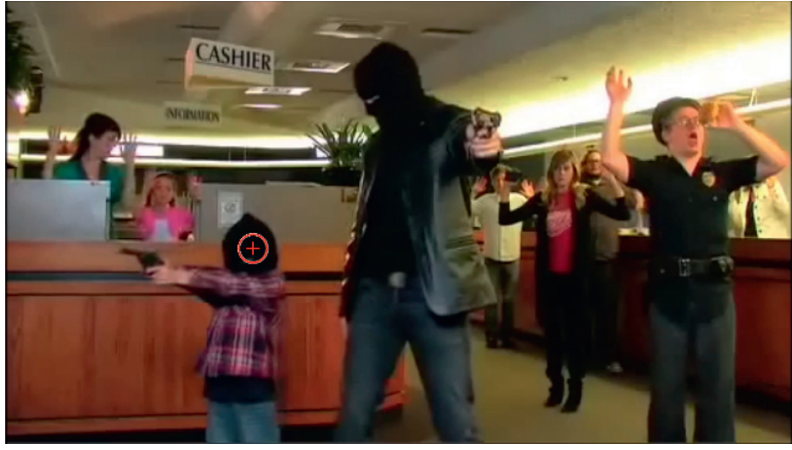

(a)

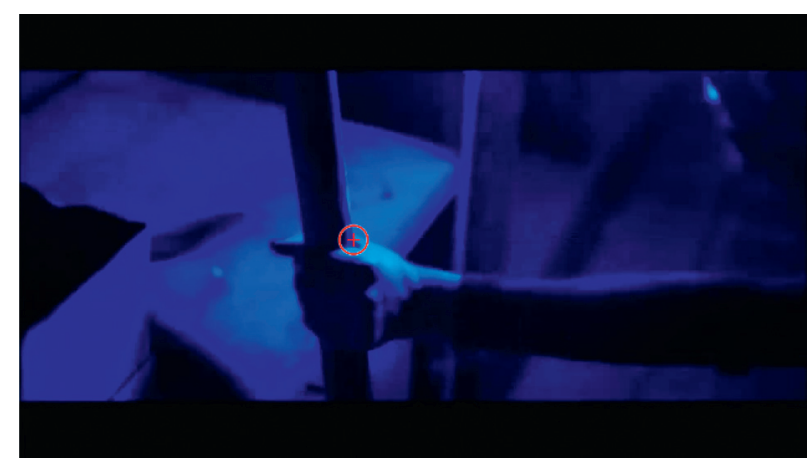

(b)

Figure 7: Two sample frames from movies with one participant's gaze positions.

The average frame's luminance was 0.4 for the left frame and 0.19 for the right one. The luminance in the neighbourhood of the gaze positions was 0.08 and 0.71 , respectively. In both cases, the average luminance of the frame and the luminance of the neighbourhood of the gaze positions are significantly different from each other. In the frame on the left, the participant looked at one of the darker points, while, in the frame on the right, he looked at a bright point. One, however, cannot ignore the luminance of the rest of the frame, because it is also important, especially when there is no other light source during the presentation of the movies or when the light in the room is very dim. Figure 8 shows the changes in the pupil diameter as determined using both of the abovementioned methods. The correlation between the pupil diameter determined by the average luminance of the entire frame and using the luminance in the neighbourhood of the gaze position was 0.87 . A very strong correlation indicates that both methods work similarly. For each participant, the correlation of the mean pupil diameter for each movie, related to one emotion with the average value of the movie luminance (i.e., without removing the influence of luminance), was also calculated. The same correlations were calculated after removing the influence of luminance (by using the average luminance of the entire frame). The median of correlation values calculated for all participants was -0.84 in the first case and -0.30 in the second case. The large difference in these values indicates that the method proposed by us for removing the effect of luminance is sufficiently effective.

In $[28,30,38]$, attempts were made to use eyetracking to recognize emotions caused by movies. However, these works did not consider the possible impact of movie dynamics on eye-tracking features. The features discussed in this article, which are associated with eye movements, may also be related to factors other than emotions. Hence, we tested the relationship of the eye-tracking features with the dynamics of the presented movies. Movies can be characterized by frequent or rare scene changes and moving or static objects. Eye movements such as fixation and saccades depend largely on the dynamics of the movie scene. This impact cannot be ignored when analyzing the signal for emotion recognition. We calculated a $D$ index describing the dynamics of the video clip

$$
D_{i}=\sum_{j}^{N} \sum_{k}^{M}\left(F_{i+1}-F_{i}\right),
$$

where $F$ is the matrix representing video frame of size $N \times$ $M$ in grayscale and $i$ is the frame number. Figure 9 depicts the variation of the $D$ index for each of the movies shown. Thus, each movie was rated for the speed at which scenes or objects changed between frames.

The obtained classification results indicate that the most difficult emotions to distinguish were negative, highly stimulating emotions from neutral, low stimulating ones (class C2 vs. C3). These two classes contain two pairs of movies: \#3 and \#18 with \#13 and \#20. According to the calculated dynamics index, these movies are characterized by small dynamics. Their dynamics are definitely smaller than those of movies \#2 and \#12 in class C1. The above tendency may indicate that the classification result, to some extent, also depends on movie dynamics and not just only on its emotional content. The distinction between the two classes of movies of similar dynamics seems to be more difficult than that between the movies of different dynamics. To test the effect of video dynamics on the obtained results, a correlation of the coefficient $D$ with the used features, averaged for all participants, was calculated. The correlations are shown in Table 10. We obtained the highest values of the average correlation $(\geq 0.4)$ for the following features: \#1-number of fixations, \#13-skewness of the saccade durations, and \#15-average pupil diameter. The ANOVA test showed that the average pupil diameter and skewness of the saccade durations were identified as statistically significant for emotion recognition (Table 8). This may indicate a relationship between the effectiveness of emotion recognition and movie dynamics. The correlation between the participants' ratings of movies used in the experiment with their dynamics was 0.45 for the arousal. These values are statistically significant and may indicate that stimulating videos are characterized by high dynamics $(\# 2, \# 12, \# 15, \# 19$, and $\# 21$ ) and nonstimulating videos by low dynamics (\#1, $\# 3$, \#14, and \#18). Dependence of arousal parameter on the movie dynamics is shown in Figure 10. 


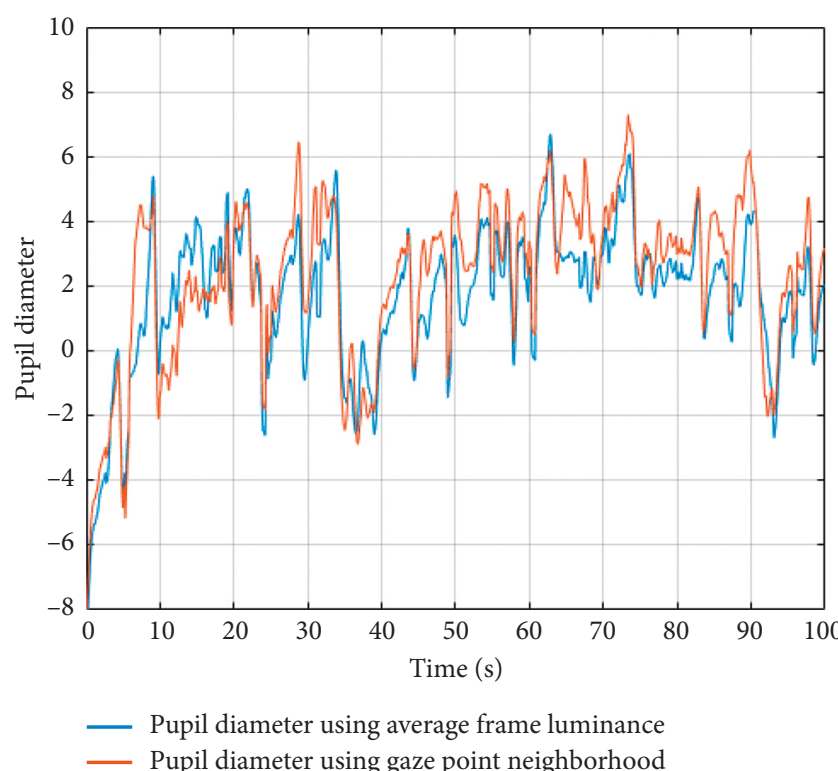

FIGURE 8: Changes in pupil eye diameter of one of the participants after removing the influence of luminance calculated using two methods.

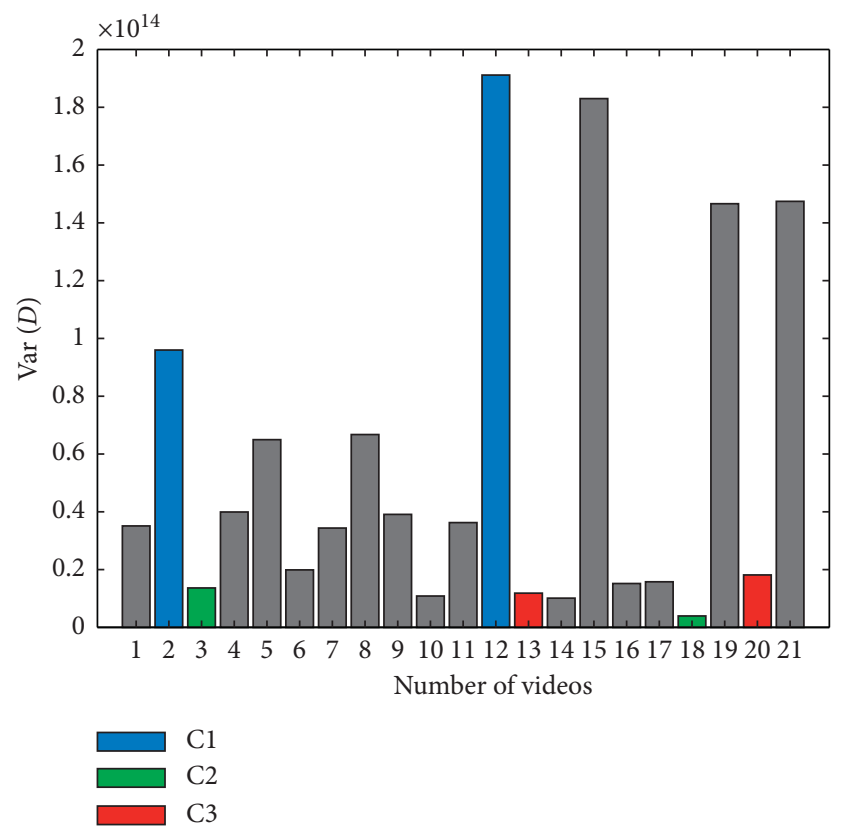

Figure 9: Movie dynamics index.

Our database contains only a few specific movies that are stimulating and not dynamic. These are, for example, movies showing finger amputation (movie \#20) or tooth extraction (movie \#10). In these movies, the camera does not change its position, and the scenes are static. Therefore, the dynamic index for these movies is low. These movies are rated as stimulating because they cause strong negative emotions related to disgust. Movies \#4 and \#17 depict horror scenes. After a few static scenes (low dynamics), terrible characters appear in these movies, thereby causing high arousal.
TABLE 10: Correlation of the coefficient $D$ with the used features averaged for all participants.

\begin{tabular}{lcc}
\hline No. & Feature & Movie dynamics \\
\hline 1 & Number of fixations & 0.43 \\
2 & Overall fixation vector & -0.34 \\
3 & Average duration of fixation & -0.32 \\
4 & Variation of fixation durations & -0.38 \\
5 & Skewness of fixation durations & 0.28 \\
6 & Kurtosis of fixation durations & 0.30 \\
7 & Average amplitude of the saccades & -0.35 \\
8 & Variation of saccade amplitudes & -0.30 \\
9 & Skewness of saccade amplitudes & -0.10 \\
10 & Kurtosis of saccade amplitudes & -0.17 \\
11 & Average duration of saccades & 0.07 \\
12 & Variance of the saccade durations & 0.10 \\
13 & Skewness of the saccade durations & 0.40 \\
14 & Kurtosis of the saccade durations & 0.39 \\
15 & Average pupil diameter & 0.62 \\
16 & Pupil diameter variance & 0.13 \\
17 & Pupil diameter skewness & 0.16 \\
18 & Pupil diameter kurtosis & 0.25 \\
\hline
\end{tabular}

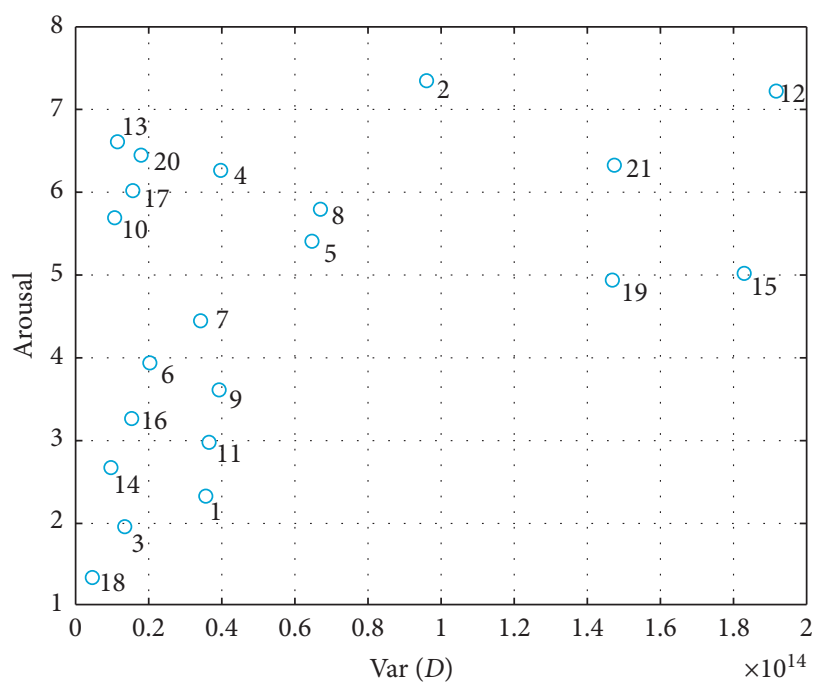

FIGURE 10: Dependence of arousal parameter on the movie dynamics.

The weakness of the work is the possible impact of movie dynamics on the results achieved. However, when recognizing the emotions evoked by movies with similar dynamics, a satisfactory classification accuracy of $78 \%$ was achieved using the LDA classifier. In the future, it is worth conducting additional research on the impact of movie dynamics.

The obtained results were compared with the results from other publications in which eye-tracking was used. However, it should be borne in mind that accurate comparison is difficult because each study used a different way of evoking emotions, a different set of eye-tracking features, and different research group. The comparison is presented in Table 11. The conducted comparison indicates that the results obtained by us are slightly better than those in similar works. The choice of a narrow group taking part in the 
TABLE 11: Comparison of our results with other studies.

\begin{tabular}{|c|c|c|c|}
\hline Works & Classes & $\begin{array}{l}\text { User-independent } \\
\text { method }\end{array}$ & $\begin{array}{l}\text { Accuracy } \\
(\%)\end{array}$ \\
\hline Our research & $\begin{array}{c}3 \text { (high arousal and high valence, high arousal and low valence, low arousal and } \\
\text { moderate valence) }\end{array}$ & + & 80.00 \\
\hline $\begin{array}{l}\text { Soleymani et al. } \\
\text { [57] }\end{array}$ & $3($ calm, medium aroused, and activated $)$ & + & 71.10 \\
\hline $\begin{array}{l}\text { Soleymani et al. } \\
\text { [57] }\end{array}$ & 3 (unpleasant, neutral, and pleasant) & + & 66.60 \\
\hline Zheng et al. [30] & 4 (sad, feeling fear, happy, and neutral) & - & 67.82 \\
\hline Lu et al. [28] & 3 (positive, neutral, and negative) & - & 77.80 \\
\hline $\begin{array}{l}\text { Soleymani et al. } \\
{[38]}\end{array}$ & 3 (calm, medium aroused, and excited) & + & 63.50 \\
\hline $\begin{array}{l}\text { Soleymani et al. } \\
\text { [38] }\end{array}$ & 3 (unpleasant, neutral, and pleasant) & + & 68.80 \\
\hline Zhao et al. [31] & 5 (happy, sad, feeling fear, disgusted, and neutral) & - & 59.81 \\
\hline
\end{tabular}

experiment (young men) may affect the result. It is possible that, in such a coherent group of people, emotions were evoked more precisely.

\section{Conclusions}

The classification results confirm the ability of recognizing emotions using eye movement features and pupil diameter. The use of an eye-tracking requires, however, the elimination of factors that may affect this classification. These factors include the effect of luminance on changes in the pupil diameter. It should be ensured that the lighting conditions remain the same throughout the experiment. The influence of luminance of the presented materials should also be compensated. This could be achieved by implementing appropriate methods, such as regression or principal component analysis.

Another factor that should be considered is the dynamics of the presented material. It seems that the dynamics of the movie can affect the accuracy of the emotion classification using the eye-tracking features. On the other hand, the dynamics of movie is related in some way to felt emotions. Research shows that high-dynamic movies have a stimulating effect. When recognizing the emotions evoked by movies with similar dynamics, a satisfactory classification accuracy of $78 \%$ using LDA was achieved. During recognition of three classes of emotions, we obtained the maximum classification accuracy of $80 \%$. It is worth emphasizing that all results were obtained using the leave-one-subject-out validation method. This implies that the presented user-independent method of emotion classification, based on eye-tracking features, can be successfully used in practice.

\section{Data Availability}

The data used to support the findings of this study are available from the corresponding author upon request.

\section{Conflicts of Interest}

The authors declare that there are no conflicts of interest regarding the publication of this paper.

\section{Acknowledgments}

This work was supported by the statutory activity of Electrical Faculty at Warsaw University of Technology under Dean's Grant in 2017.

\section{References}

[1] E. Bal, E. Harden, D. Lamb, A. V. Van Hecke, J. W. Denver, and S. W. Porges, "Emotion recognition in children with autism spectrum disorders: relations to eye gaze and autonomic state," Journal of Autism and Developmental Disorders, vol. 40, no. 3, pp. 358-370, 2010.

[2] S. B. Daily, M. T. James, D. Cherry et al., "Affective computing: historical foundations, current applications, and future trends," in Emotions and Affect in Human Factors and Human-Computer Interaction, pp. 213-231, Elsevier Academic Press, San Diego, CA, USA, 2017.

[3] K. Karpouzis and G. N. Yannakakis, Emotion in Games, Vol. 4, Springer International Publishing, Cham, Switzerland, 2016.

[4] C. Holmgård, G. N. Yannakakis, K.-I. Karstoft, and H. S. Andersen, "Stress detection for PTSD via the StartleMart game," in Proceedings of the 2013 Humaine Association Conference on Affective Computing and Intelligent Interaction, pp. 523-528, Geneva, Switzerland, September 2013.

[5] A. Pampouchidou, P. G. Simos, K. Marias et al., "Automatic assessment of depression based on visual cues: a systematic review," IEEE Transactions on Affective Computing, vol. 10, no. 4, pp. 445-470, 2019.

[6] B. Li, "A facial affect analysis system for autism spectrum disorder," in Proceedings of the 2019 IEEE International Conference on Image Processing (ICIP), pp. 4549-4553, Taipei, Taiwan, September 2019.

[7] S. Sarabadani, L. C. Schudlo, A.-A. Samadani, and A. Kushki, "Physiological detection of affective states in children with autism spectrum disorder," IEEE Transactions on Affective Computing, p. 1, 2018.

[8] L. Cen, F. Wu, Z. L. Yu, and F. Hu, "A real-ime speech emotion recognition system and its application in online learning," in Emotions, Technology, Design, and Learning, S. Y. Tettegah and M. Gartmeier, Eds., pp. 27-46, Academic Press, San Diego, DA, USA, 2016.

[9] S. S. S. Darnell, "EngageME," in Proceedings of the Extended Abstracts of the 32nd Annual ACM Conference on Human 
Factors in Computing Systems - CHI EA '14, pp. 359-362, Toronto, Canada, 2014.

[10] M. Soleymani, M. Larson, T. Pun, and A. Hanjalic, "Corpus development for affective video indexing," 2012, https://arxiv. org/abs/1211.5492.

[11] Y. Ding, X. Hu, Z. Xia, Y.-J. Liu, and D. Zhang, "Inter-brain EEG feature extraction and analysis for continuous implicit emotion tagging during video watching," IEEE Transactions on Affective Computing, p. 1, 2018.

[12] M. Soleymani, S. Asghari-Esfeden, Y. Fu, and M. Pantic, "Analysis of EEG signals and facial expressions for continuous emotion detection," IEEE Transactions on Affective Computing, vol. 7, no. 1, pp. 17-28, 2016.

[13] S. Koelstra and I. Patras, "Fusion of facial expressions and EEG for implicit affective tagging," Image and Vision Computing, vol. 31, no. 2, pp. 164-174, 2013.

[14] P. Tarnowski, M. Kołodziej, A. Majkowski, and R. J. Rak, "Emotion recognition using facial expressions," Procedia Computer Science, vol. 108, pp. 1175-1184, 2017.

[15] P. Tarnowski, M. Kolodziej, A. Majkowski, and R. J. Rak, "“Anthropometric facial features in emotion recognition," in Proceedings of 2018 19th International Conference Computational Problems of Electrical Engineering, CPEE 2018, Banska Stiavnica, Slovakia, September 2018.

[16] W. Dai, D. Han, Y. Dai, and D. Xu, "Emotion recognition and affective computing on vocal social media," Information \& Management, vol. 52, no. 7, pp. 777-788, 2015.

[17] A. Majkowski, M. Kolodziej, R. J. Rak, and R. Korczyński, "Classification of emotions from speech signal," in Proceedings of the 2016 Signal Processing: Algorithms, Architectures, Arrangements, and Applications (SPA), pp. 276-281, Poznan, Poland, September 2016.

[18] J. Han, X. Ji, X. Hu, L. Guo, and T. Liu, "Arousal recognition using audio-visual features and FMRI-based brain response," IEEE Transactions on Affective Computing, vol. 6, no. 4, pp. 337-347, 2015.

[19] A. Goshvarpour, A. Abbasi, and A. Goshvarpour, "An accurate emotion recognition system using ECG and GSR signals and matching pursuit method," Biomedical Journal, vol. 40, no. 6, pp. 355-368, 2017.

[20] P. Das, A. Khasnobish, and D. N. Tibarewala, "Emotion recognition employing ECG and GSR signals as markers of ANS," in Proceedings of the 2016 Conference on Advances in Signal Processing (CASP), pp. 37-42, Pune, India, June 2016.

[21] Y.-L. Hsu, J.-S. Wang, W.-C. Chiang, and C.-H. Hung, "Automatic ECG-based emotion recognition in music listening," IEEE Transactions on Affective Computing, vol. 11, no. 1, pp. 85-99, 2017.

[22] V. Kehri, R. Ingle, S. Patil, and R. N. Awale, "Analysis of facial EMG signal for emotion recognition using wavelet packet transform and SVM," in Proceedings of the Machine Intelligence and Signal Analysis, pp. 247-257, Singapore, 2019.

[23] X. Sun, T. Hong, C. Li, and F. Ren, "Hybrid spatiotemporal models for sentiment classification via galvanic skin response," Neurocomputing, vol. 358, pp. 385-400, 2019.

[24] S. Hassani, I. Bafadel, A. Bekhatro, E. Al Blooshi, S. Ahmed, and M. Alahmad, "Physiological signal-based emotion recognition system," in Proceedings of the 2017 4th IEEE International Conference on Engineering Technologies and Applied Sciences (ICETAS), pp. 1-5, Salmabad, Bahrain, November 2017.

[25] R. Rakshit, V. R. Reddy, and P. Deshpande, "Emotion detection and recognition using HRV features derived from photoplethysmogram signals," in Proceedings of the 2nd workshop on Emotion Representations and Modelling for Companion Systems, pp. 1-6, Tokyo, Japan, November 2016.

[26] J. A. Domínguez-Jiménez, K. C. Campo-Landines, J. C. Martínez-Santos, E. J. Delahoz, and S. H. ContrerasOrtiz, "A machine learning model for emotion recognition from physiological signals," Biomedical Signal Processing and Control, vol. 55, Article ID 101646, 2020.

[27] L. Mirmohamadsadeghi, A. Yazdani, and J.-M. Vesin, "Using cardio-respiratory signals to recognize emotions elicited by watching music video clips," in Proceedings of the 2016 IEEE 18th International Workshop on Multimedia Signal Processing (MMSP), Montreal, Canada, September 2016.

[28] Y. Lu, W.-L. Zheng, B. Li, and B.-L. Lu, "Combining eye movements and EEG to enhance emotion recognition," in Proceedings of the 24th International Conference on Artificial Intelligence, pp. 1170-1176, Buenos Aires, Argentina, 2015.

[29] W. L. Zheng, B. N. Dong, and B. L. Lu, "Multimodal emotion recognition using EEG and eye tracking data," in Proceedings of the 2014 36th Annual International Conference of the IEEE Engineering in Medicine and Biology Society, pp. 5040-5043, Chicago, IL, USA, August 2014.

[30] W.-L. Zheng, W. Liu, Y. Lu, B.-L. Lu, and A. Cichocki, "EmotionMeter: a multimodal framework for recognizing human emotions," IEEE Transactions on Cybernetics, vol. 49, no. 3, pp. 1110-1122, 2019.

[31] L.-M. Zhao, R. Li, W.-L. Zheng, and B.-L. Lu, "Classification of five emotions from EEG and eye movement signals: complementary representation properties," in Proceedings of the 2019 9th International IEEE/EMBS Conference on Neural Engineering (NER), pp. 611-614, San Francisco, CA, USA, March 2019.

[32] J. Z. Lim, J. Mountstephens, and J. Teo, "Emotion recognition using eye-tracking: taxonomy, review and current challenges," Sensors, vol. 20, no. 8, p. 2384, 2020.

[33] T. Zhang, A. El Ali, C. Wang, X. Zhu, and P. Cesar, "CorrFeat: correlation-based feature extraction algorithm using skin conductance and pupil diameter for emotion recognition," in Proceedings of the 2019 International Conference on Multimodal Interaction, pp. 404-408, Suzhou, China, October 2019.

[34] M. M. Bradley, L. Miccoli, M. A. Escrig, and P. J. Lang, "The pupil as a measure of emotional arousal and autonomic activation," Psychophysiology, vol. 45, no. 4, pp. 602-607, 2008.

[35] P. Raiturkar, A. Kleinsmith, A. Keil, A. Banerjee, and E. Jain, "Decoupling light reflex from pupillary dilation to measure emotional arousal in videos," in Proceedings of the ACM Symposium on Applied Perception, pp. 89-96, Anaheim, CA, USA, July 2016.

[36] T. Partala and V. Surakka, "Pupil size variation as an indication of affective processing," International Journal of $\mathrm{Hu}$ man-Computer Studies, vol. 59, no. 1-2, pp. 185-198, 2003.

[37] K. Holmqvist, M. Nyström, R. Anderson, R. Dewhurst, H. Jarodzka, and J. van der Weijer, Eye Tracking: A Comprehensive Guide to Methods and Measures, Oxford University Press, New York, NY, USA, 2011.

[38] M. Soleymani, J. Lichtenauer, T. Pun, and M. Pantic, "A multimodal database for affect recognition and implicit tagging," IEEE Transactions on Affective Computing, vol. 3, no. 1, pp. 42-55, 2012.

[39] E. H. Hess and J. M. Polt, "Pupil size as related to interest value of visual stimuli," Science, vol. 132, no. 3423, pp. 349-350, 1960.

[40] W. L. Libby, B. C. Lacey, and J. I. Lacey, "Pupillary and cardiac activity during visual attention," Psychophysiology, vol. 10, no. 3, pp. 270-294, 1973. 
[41] A. Alhargan, N. Cooke, and T. Binjammaz, "Affect recognition in an interactive gaming environment using eye tracking," in Proceedings of the 2017 Seventh International Conference on Affective Computing and Intelligent Interaction (ACII), pp. 285-291, San Antonio, TX, USA, October 2017.

[42] S. Koelstra, C. Muhl, M. Soleymani et al., "DEAP: a database for emotion analysis ;using physiological signals," IEEE Transactions on Affective Computing, vol. 3, no. 1, pp. 18-31, 2012.

[43] A. G. Money and H. Agius, "Analysing user physiological responses for affective video summarisation," Displays, vol. 30, no. 2, pp. 59-70, 2009.

[44] M. Bradley and P. Lang, "The international affective digitized sounds (2nd edition; IADS-2): affective ratings of sounds and instruction manual," Technical report B-3, University of Florida, Gainesville, FL, USA, 2007.

[45] P. Ekman, W. V. Friesen, M. O'Sullivan et al., "Universals and cultural differences in the judgments of facial expressions of emotion," Journal of Personality and Social Psychology, vol. 53, no. 4, pp. 712-717, Oct. 1987.

[46] J. Posner, J. A. Russell, and B. S. Peterson, “The circumplex model of affect: an integrative approach to affective neuroscience, cognitive development, and psychopathology," Development and Psychopathology, vol. 17, no. 3, pp. 715-734, 2005.

[47] Y. Nakayama, Y. Takano, M. Matsubara, K. Suzuki, and H. Terasawa, "The sound of smile: auditory biofeedback of facial EMG activity," Displays, vol. 47, pp. 32-39, 2017.

[48] J. A. Coan and J. J. B. Allen, Handbook of Emotion Elicitation and Assessment, Oxford University Press, New York, NY, USA, 2007.

[49] R. Dietz and A. Lang, “Æffective agents - Dietz \& Lang," in Proceedings of the Third International Cognitive Technology Conference, San Francisco, CA, USA, 1999.

[50] M. Horvat, M. Vuković, and Ž. Car, "Evaluation of keyword search in affective multimedia databases," Evaluation of keyword search in affective multimedia databases," in Transactions on Computational Collective Intelligence XXI: Special Issue on Keyword Search and Big Data, N. T. Nguyen, R. Kowalczyk, and P. Rupino da Cunha, Eds., pp. 50-68, Springer Berlin Heidelberg, Berlin, Heidelberg, 2016.

[51] P. Tarnowski, M. Kołodziej, A. Majkowski, and R. J. Rak, “A system for synchronous acquisition of selected physiological signals aimed at emotion recognition," Przeglad Elektrotechniczny, vol. 1, no. 12, pp. 329-333, 2016.

[52] "Basics eyetribe-docs," https://theeyetribe.com/dev.theeyetribe. com/dev.theeyetribe.com/general/index.html.

[53] D. D. Salvucci and J. H. Goldberg, "Identifying fixations and saccades in eye-tracking protocols," in Proceedings of the 2000 Symposium on Eye Tracking Research \& Applications, pp. 71-78, New York, NY, USA, 2000.

[54] C.-F. Chi and F.-T. Lin, "A new method for describing search patterns and quantifying visual load using eye movement data," International Journal of Industrial Ergonomics, vol. 19, no. 3, pp. 249-257, 1997.

[55] M. R. Ogiela and R. Tadeusiewicz, "Modern computational intelligence methods for the interpretation of medical images, in Studies in Computational Intelligence," vol. 84, Springer, Berlin, Germany, 2008.

[56] C. J. Ellis, "The pupillary light reflex in normal subjects," British Journal of Ophthalmology, vol. 65, no. 11, pp. 754-759, 1981.
[57] M. Soleymani, M. Pantic, and T. Pun, "Multimodal emotion recognition in response to videos," IEEE Transactions on Affective Computing, vol. 3, no. 2, pp. 211-223, 2012. 